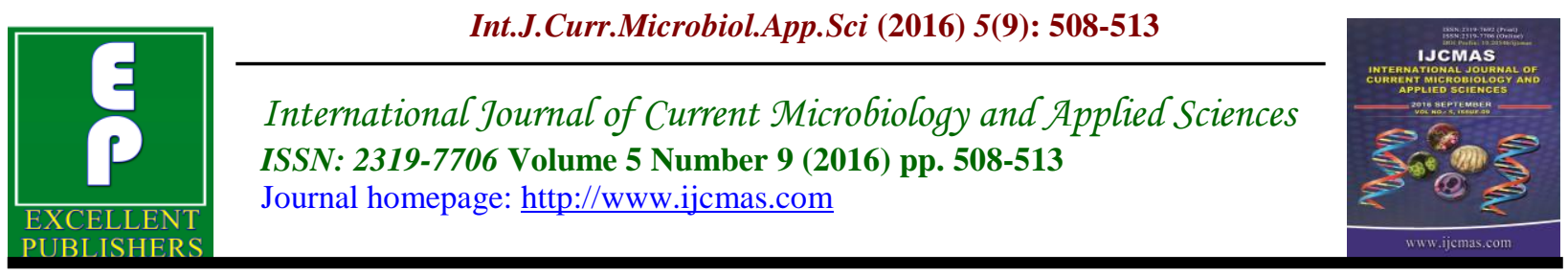

Original Research Article

http://dx.doi.org/10.20546/ijcmas.2016.509.056

\title{
Microbial Contamination of Mobile Phones in a Tertiary Health Care Setting
}

\author{
J. Lavanya ${ }^{1 *}$, N.B. Swaroop Rani ${ }^{1}$, Manoj Jais ${ }^{2}$ and Amruth Kishan Upadhya ${ }^{1}$ \\ ${ }^{1}$ Subbaiah Institute of Medical Sciences, Shimoga, Karnataka State, India \\ ${ }^{2}$ Lady Hardinge Medical College, New Delhi, India
}

*Corresponding author

\section{Keywords \\ Mobile phones, contamination, pediatric ICU, CoNS, health care workers.}

\begin{tabular}{l}
\hline Article Info \\
\hline Accepted: \\
20 August 2016 \\
Available Online: \\
10 September 2016
\end{tabular}

\section{A B S T R A C T}

Mobile phones are widely used as nonmedical portable electronic devices and it is in close contact with the body. This study shows how mobile phones can pose serious threats to patients in Paediatric ICU and nursery. 100 samples from keypads of mobile phones were taken using moistened sterile swab from doctors and nurses working in pediatric intensive care unit and nursery of lady hardinge medical college and associated hospitals, New delhi. Bacteria were identified and antibiotic sensitivity determined using standard procedures. $56 \%$ of mobile phones showed growth. Majority of isolates were CoNS [Coagulase negative Staphylococci] (71.4\%), followed by S.aureus (10.7\%), Acinetobacter (10.7\%) and E.coli (7.1\%). $45 \%$ CoNS and $33.3 \%$ S.aureus were resistant to penicillin, oxacillin, gentamicin, ciprofloxacin, erythromycin, Cotrimoxazole and clindamycin. 7.5\% CoNS and $16.6 \% \mathrm{~S}$. aureus were resistant to levofloxacin and tetracycline. $33.3 \%$ Acinetobacter and $25 \% \mathrm{E}$. coli were resistant to gentamicin, ciprofloxacin, erythromycin cotrimoxazole and ceftazidime, whereas only $16.6 \%$ Acinetobacter were resistant to levofloxacin. This study provides the baseline data for formulating strict guidelines to minimize cross-infection by means of mobile phones in pediatric ICU and nursery.

\section{Introduction}

Health care associated infections (HCAI) can be defined as: An infection occurring in a patient in a hospital or other health care facility in whom the infection was not present or incubating at the time of admission. This includes infections acquired in the hospital but appearing after discharge, and also occupational infections among staff of the facility (World Health Organization, 2002). The term healthcare-associated infections (HCAI) replaced old ones such as nosocomial, hospital-acquired or hospitalonset infections, as a better reflection of the diverse healthcare settings currently available to patients (Pittet et al., 1999).

Health care associated infections are associated with significant morbidity, mortality \& health care costs particularly in ICU setting. Organisms causing particular concern include Methicillin resistant Staphylococcus aureus [MRSA], multi-drug 
resistant Pseudomonas, \& Vancomycin resistant Enterococci.

Paediatric ICU and paediatric nursery are the workplaces that need highest hygienic standards, also the same requirements for the personnel working there and the equipment used by them. Mobile phones are widely used as nonmedical portable electronic devices and it is in close contact with the body. It is used for communication by health care workers in every location including ICU but not cleaned properly, as they do not wash their hands as often as they should (Ulger et al., 2009). In a study it was discovered that the average cell phone is dirtier than either a toilet seat or the bottom of the shoe (Arora et al., 2009).

Today India has 287 million mobile phone users and this account for $85 \%$ of all the communications users. Experts indicate that in 2005 there were more than 6.7 billion telephone users worldwide. In the health care setting, they are essential for quick and easy access to laboratory and imaging results, for consultations, and sometimes for life threatening emergencies (Akinyemi et al., 2009).

These can be put in vibratory mode in intensive care units, post-operative wards and operation theatres etc. however they are seldom cleaned and are often touched during or after examination of patients and handling of specimens without proper hand washing. These cell phones can harbour various potential pathogens and become an exogenous source of nosocomial infection among hospitalized patients and also a potential health hazard for self and family members.

This study is designed to find out the dynamics of bacterial contamination of mobile phones of health care workers. Study findings should help us evaluate the role of health care workers in cross transmission and its impact on the patients admitted in PICU and paediatric nursery, ultimately improving hand cleansing and disinfection practices.

\section{Materials and Methods}

This study was conducted in the paediatric ICU and Nursery of Lady Hardinge Medical College and Associated Hospitals, New Delhi

\section{Sampling and sample size}

Samples from mobile phones of 100 resident doctors and nursing staff posted in Paediatric intensive care unit and nursery of Lady Hardinge Medical College and Associated Hospitals.

\section{Methodology}

Informed consent was obtained from each participant.

Confidentiality was maintained about the identity of participants.

Instead each participant was given id code.

In between patient care, before hand washing samples were obtained.

Samples were taken using sterile swab moistened with saline from key pad of mobile phones.

\section{Culture Methods}

Collected samples were immediately cultured on blood agar and Mac conkey agar plates using calibrated loop.

Cultured plates were immediately transported to laboratory and kept for overnight incubation at $370 \mathrm{C}$. 
The media were examined for any growth at 24 hrs. The obtained growth was subjected to identification based on standard bacteriological procedures like colony morphology on blood and Mac conkey agar plates, Gram's staining and biochemical reactions. Further, antibiotic sensitivity testing of isolated organisms was done using Kirby bauer disc diffusion method.

\section{Results and Discussion}

$56 \%$ of mobile phones showed growth.

In our study, $56 \%$ of mobile phones $(n=100)$ used by doctors and nursing staff in pediatric ICU and nursery showed bacterial growth.

Usha arora et al., (2009), Kabir O. Akinyemi (2009) and Nilima Tankhiwale (2012) reported that $40.6 \%, 62 \%$ and $65 \%$ of doctors and other health care staff's mobile phones were contaminated by bacterial pathogens respectively which corelates with our study findings (Tankhiwale et al., 2012; Karabay et al., 2007).

Behaviour of HCWs contributing to bacterial growth on mobile phones is:

1. Infrequent cleaning of mobile phones during patient care.

2. Some doctors are unaware of the fact that mobile phones can also act as the vector for transmission of pathogenic organisms to patients.

3. Most of them who are aware of its pathogenic potential also don't clean, as per the instructions of mobile phone manufacturers that contact with water or liquid disinfectant might damage the software of their expensive mobile phones.
Table 1 shows that Majority of isolates were CoNS (71.4\%), followed by S.aureus (10.7\%), Acinetobacter (10.7\%) and E.coli (7.1\%).

Kabir O. Akinyemi et al., and Karabay et al., also reported CoNS as the most prevalent bacterial agent isolated from mobile phones at the rate of $42.7 \%$ and $68.4 \%$ respectively. Other organisms isolated by them were Staphylococcus aureus, E.coli, Acinetobacter and other pathogenic organisms encountered in health care associated infections (Tambekar et al., 2008; Jayalakshmi et al., 2008).

Thus in all the above studies, CoNS were found to be the most commonly isolated organism similar to our study. Even though CoNS is considered as a resident flora, it can acquire the characteristics of patients' flora and become pathogenic. This is a wellknown fact that organisms like S.aureus and CoNS resist drying and thus can survive and multiply rapidly in the warm environments like cell phones. CoNS are the most commonly isolated organism in blood stream infections and surgical site infections, so has a major impact on patients' health.

Other organisms isolated were S.aureus, Acinetobacter and E.coli which are known to play significant role in health care associated infections. S.aureus is implicated as a major pathogen in blood stream infections, pneumonia as well as in surgical site infections. E.coli is the most commonly isolated organism from urinary tract infections. The ability of Acinetobacter to contaminate cell phones is quite possible as this is a multi-drug resistant water and soil organism and is responsible for infection in predisposed patients in the hospital. In our study, bacterial isolates from mobile phones were similar to those isolated from hands of health care workers. 
This shows that bacteria present on contaminated hands could be easily transferred to mobile phones of health care workers, which are frequently touched during patient care.

In the present study, $45 \%$ CoNS and $33.3 \%$ S.aureus were resistant to penicillin, oxacillin, gentamicin, ciprofloxacin, erythromycin, cotrimoxazole and clindamycin. $7.5 \% \mathrm{CoNS}$ and $16.6 \% \mathrm{~S}$. aureus were resistant to levofloxacin and tetracycline. 33.3\% Acinetobacter and 25\% $E$. coli were resistant to gentamicin, ciprofloxacin, erythromycin cotrimoxazole and ceftazidime, whereas only $16.6 \%$ Acinetobacter were resistant to levofloxacin.

Table.1 Isolates from mobile phones

\begin{tabular}{|l|l|l|l|}
\hline Sl.no & Bacterial Pathogen & No. of Isolates $(\mathbf{N}=56)$ & Percentage \\
\hline 1 & CoNS & 40 & $71.4 \%$ \\
\hline 2 & S.aureus & 6 & $10.7 \%$ \\
\hline 3 & Acinetobacter $s p p$ & 6 & $10.7 \%$ \\
\hline 4 & E.coli & 4 & $7.1 \%$ \\
\hline
\end{tabular}

Table.2 Antibiotic resistance pattern of bacterial isolates from mobile phones

\begin{tabular}{|c|c|c|c|c|}
\hline Antibiotics & CoNS (N=40) & S. aureus $(\mathbf{N}=\mathbf{6})$ & Acinetobacter $\mathbf{( N = 6 )}$ & E.coli $(\mathbf{N}=\mathbf{4})$ \\
\hline P & $18(45 \%)$ & $2(33.3 \%)$ & - & - \\
\hline OX & $18(45 \%)$ & $2(33.3 \%)$ & - & - \\
\hline G & $18(45 \%)$ & $2(33.3 \%)$ & $2(33.3 \%)$ & $1(25 \%)$ \\
\hline CF & $18(45 \%)$ & $2(33.3 \%)$ & $2(33.3 \%)$ & $1(25 \%)$ \\
\hline LE & $3(7.5 \%)$ & $1(16.6 \%)$ & $1(16.6 \%)$ & - \\
\hline E & $18(45 \%)$ & $2(33.3 \%)$ & $2(33.3 \%)$ & $1(25 \%)$ \\
\hline CO & $18(45 \%)$ & $2(33.3 \%)$ & $2(33.3 \%)$ & $1(25 \%)$ \\
\hline CD & $18(45 \%)$ & $2(33.3 \%)$ & - & - \\
\hline T & $3(7.5 \%)$ & $1(16.6 \%)$ & - & - \\
\hline CA & - & - & $2(33.3 \%)$ & $1(25 \%)$ \\
\hline PIT & - & - & $1(16.6 \%)$ & $1(25 \%)$ \\
\hline I & - & - & $2(33.3 \%)$ & $1(25 \%)$ \\
\hline MRP & - & - & $1(16.6 \%)$ & $0 \%$ \\
\hline TIG & - & - & $0 \%$ & $0 \%$ \\
\hline TE & $0 \%$ & $0 \%$ & - & - \\
\hline CL & - & - & $0 \%$ & $0 \%$ \\
\hline LZ & $0 \%$ & $0 \%$ & - & - \\
\hline NT & - & - & $1(16.6 \%)$ & $0 \%$ \\
\hline AK & - & - & $2(33.3 \%)$ & $1(25 \%)$ \\
\hline
\end{tabular}

Thus, the isolated organisms were resistant to most of the commonly used drugs in clinical settings. The isolation of MRSA and multidrug resistant Acinetobacter has been the matter of concern. This proves the pathogenic potential of the organisms that are isolated from mobile phones.

Many factors are responsible for bacterial contamination of mobile phones: Mobile 
phones can be put in vibratory mode in intensive care units, post-operative wards and operation theatres etc. However they are seldom cleaned and are often touched during or after examination of patients and handling of specimens without proper hand washing.

Brady et al., reported that the combination of constant handling and heat generated by the phones creates favorable condition for multiplication of organisms that are normally found in our skin. Thus mobile phones are the perfect breeding sites for most of the pathogenic organisms encountered in health care associated infections (Brady et al., 2006).

In our study contamination of mobile phones could be due to many reasons. Most important is low compliance of hand washing, thus bacteria that are carried by hands are transmitted to mobile phones that are usually in contact with the hands. Only hand washing does not prevent transmission of infection from mobile phones, but disinfection of mobile phones with isopropyl alcohol needs to be practiced. Otherwise bacteria present on mobile phones get transferred to clean hands and the cycle of cross infection continues.

Currently in many institutions, strict guidelines have not been implemented to restrict medical staff from carrying mobile phones into the sterile environment of the OT, ICU or other critical areas. There are also no cleaning guidelines for mobile phones of HCWs. People tried many ways to clean and sterilize their mobile phones but the best way is ultrasonic cleaning by an ultrasonic cleaner which clean the mobile phones thoroughly and safely (The best way to clean your cell phone -ultrasonic cleaning, 2010). Thus there is need for following strict infection control practices to minimize HCAI.
Restriction of using mobile phone while working hours is not the practical solution of the problem. In resource poor hospital settings like India, ultrasonic cleaner is not available at most of places. Therefore simple measures like hand washing, cleaning of mobile phones with $70 \%$ isopropyl alcohol, using hand free mobile phone while working hours, well controlled infection control plan and regular training to $\mathrm{HCW}$ are recommended to reduce the rate of health care associated infections.

\section{References}

Akinyemi, K.O., Atapu, A.D. et al. 2009. The potential role of mobile phones in the spread of bacterial infections. $J$. Infect. Dev. Ctries., 3(8): 628-32.

Arora, U., Devi, P. et al. 2009. Cell phones a modern stay house for bacterial pathogens. J. Med. Edu. Res., Vol 11, No. 3.

Brady, R.R., Wasson, A., Stirling, I., McAllister, C., Damani, N.N. 2006. Is your phone bugged? The incidence of bacteria known to cause nosocomial infection in healthcare workers mobile phones. J. Hosp. Infect., 62: 123-125.

Jayalakshmi, J., Appalaraju, B., Usha, S. 2008. Cellphones as reservoirs of nosocomial pathogens. J. Assoc. Physicians India, 56: 388-9.

Karabay, O., Koçoglu, E. et al. 2007. The role of mobile phones in the spread of bacteria associated with nosocomial infections. J. Infect. Developing Countries, 1(1): 72-73.

Pittet, D., Dharan, S., Touveneau, S., Sauvan, V., Perneger, T.V. 1999. Bacterial contamination of the hands of hospital staff during routine patient care. Arch. Intern. Med., 26: 159(8): 821-6.

Tambekar, D.H., Gulhane, P.B., et al. 2008. Nosocomial Hazards of Doctor`s 
Mobile Phones in Hospitals. J. Med. Sci., 8: 73-76.

Tankhiwale, N., Gupta, V., et al. 2012. Nosocomial Hazards of Doctor's Mobile Phones. Indian Med. Gazette, 283-285.

The best way to clean your cell phone ultrasonic cleaning. Accessed on, Aug. 2010. Available from: http://www.articlesbase.com/healtharticles/the-best-way-to-cleanyourcell-phone-ultrasonic-cleaning1082298.html.

Ulger, F., Esen, S., Dilek, A., Yanik, K., Gunaydin, M., Leblebicioglu, H. 2009.
Are we aware how contaminated our mobile phones with nosocomial pathogens? Ann. Clin. Microbiol. Antimicrob., 6(8): $7 . \quad$ doi: 10.1186/1476-0711-8-7.

World Health Organization. 2002. Department of Communicable Disease, Surveillance and Response. Prevention of hospital-acquired infections, a practical guide. Available from:

http://www.who.int/csr/resources/publ ications/whocdscsreph200212.pdf.

\section{How to cite this article:}

Lavanya, J., N.B. Swaroop Rani, Manoj Jais and Amruth Kishan Upadhya. 2016. Microbial Contamination of Mobile Phones in a Tertiary Health Care Setting. Int.J.Curr.Microbiol.App.Sci. 5(9): 508-513. doi: http://dx.doi.org/10.20546/ijcmas.2016.509.056 